\title{
Regenerated gratings
}

John Canning

Somnath Bandyopadhyay

Michael Stevenson

Palas Biswas

Jacob Fenton

Mattias Aslund
Interdisciplinary Photonics Laboratories (iPL), School of Chemistry, University of Sydney, Australia

Fibre Optics Laboratory, Central Glass and Ceramic Research Institute (CGCRI), Council of Scientific \& Industrial Research, Kolkata - 700032, India

Interdisciplinary Photonics Laboratories (iPL), School of Chemistry, University of Sydney, Australia

Fibre Optics Laboratory, Central Glass and Ceramic Research Institute (CGCRI), Council of Scientific \& Industrial Research, Kolkata - 700032, India

Interdisciplinary Photonics Laboratories (iPL), School of Chemistry, University of Sydney, Australia

Strong regenerated gratings with a maximum grating strength exceeding (40-50) dB are fabricated inside an optical fibre by bulk macro thermal processing $\sim 900^{\circ} \mathrm{C}$ using a UV-laser seeded Bragg grating. Further annealing between $1000^{\circ} \mathrm{C}$ and $1100^{\circ} \mathrm{C}$ leads to a stabilised grating $\sim 18 \mathrm{~dB}$ in strength. This suffers no further degradation at $1100^{\circ} \mathrm{C}$ for the period monitored, over 4 hours. The potential resolution of this process is demonstrated by regenerating two complex profiles. Phase information is retained between seed and regenerated structures. This opens the way for nano-engineering of materials using thermal processing and seed templates. [D0I: 10.2971/je0s.2009.09052]

Keywords: regeneration, regenerated gratings, crystallization, gratings, sensors, Bragg grating

\section{INTRODUCTION}

Silica remains the key optoelectronic and photonic medium, the essence of nearly all modern optical transport systems. Engineering of silica in its various forms ranges from 1 to 3-dimensional waveguide and periodic structures, including recent interest in 3-D photonic crystals. Most of the processing methods involve complex vapour deposition and various codopants, which have an advantage of overcoming the lack of finesse involved with general formation of glass structure through high temperature processing and quenching. Nevertheless, to obtain micron or sub-micron precision over the processing of glass, invariably post processing methods are commonly used, ranging from etching of systems with dopants to laser processing using UV to mid IR lasers. Concrete examples of micron scale processing of glass include direct written waveguides, Bragg gratings in waveguides and optical fibres and photonic crystals. The drawback with these postprocessing techniques is that they generally produce glass that is structurally less stable than the starting phase; this includes that produced by femtosecond laser induced melting and solidification. For many applications the thermal stability of laser induced glass changes determines the limits in which they can operate; for standard telecommunications applications, type fibre Bragg gratings that can operate to $80^{\circ} \mathrm{C}$ for 25 years are required - such gratings can in principle operate for lengthy periods up to $300^{\circ} \mathrm{C}$. Gratings that can operate at temperatures well above standard telecommunication requirements are critical to the success of many real time sensing applications. In the oil and gas industries, for example, the desirable operating limit for temperature in many applications is ideally $\sim 400^{\circ} \mathrm{C}$ although standard oil bores are typically quoted as having an environment no more than $\sim 180^{\circ} \mathrm{C}-$ $250^{\circ} \mathrm{C}[1,2]$, variations can occur and the increasing depth of the next generation bores suggest sensors that can operate to $400^{\circ} \mathrm{C}$ or more are desirable for long term or permanent operation. In industries involving high temperature furnaces, such as aluminium smelting or coal based power stations, it would be of interest to be able to monitor temperatures in excess of $1000^{\circ} \mathrm{C}$. Similar temperature requirements span many other types of industries - structural health monitoring of buildings need to be able to operate in temperatures above $400^{\circ} \mathrm{C}$ in the event of a serious fire [3], engine turbines in various vehicle formats, particularly aircraft, can reach temperatures well above this, whilst integration into next generation composite structures in many of these applications needs to be carried out in temperatures above $300^{\circ} \mathrm{C}-400^{\circ} \mathrm{C}$. Within all these applications, the same stringent fabrication capabilities imposed on telecommunications are also increasingly desirable as sensor system and components become more complex than simple low reflection filters. Complex filter properties such as apodisation, chirping, phase shifts and more are increasingly in demand. Therefore, there is a need to produce high temperature gratings that retain the best features of the current workhorse, the type I grating. 
Previous studies have already established that operable temperature of FBGs can be increased by several means, including tailoring the glass composition [4,5], pre-processing with seed irradiation [6, 7], the formation of type-In (or type II $A$ ) [8][10] gratings and type-II [11, 12] gratings, including those inscribed using femtosecond IR lasers [13]. For a general review on photosensitivity and grating types, see [14]. Another variant with superior high temperature stability is the so-called "chemical composition grating (CCG)" [15] where a periodic index modulation can be regenerated after erasure of the UV induced type-I grating written in $\mathrm{H}$-loaded germanosilicate fibre, which happens to contain fluorine, if annealed $\sim 1000^{\circ} \mathrm{C}$. The prediction was a local reduction of fluorine in the UVexposed zones at that high temperature through diffusion of hydrogen fluoride. A subsequent study on annealing of typeI gratings at high temperature, however, has shown that the presence of fluorine is not necessary for this regeneration of index modulation [16]. So-called chemical composition gratings (CCG) are found in Er-doped fiber with other dopants as $\mathrm{Ge}, \mathrm{Al}$ and $\mathrm{Sn}$. Very recently, the general phenomenon of regeneration has been found in simple H-loaded germanosilicate fibre [17]. The implication of this result was soon recognised; rather than rely on a diffusive interpretation and subsequent polarisability change as the basis for writing gratings, an alternative approach to engineering the index change based on glass structural transformation arising from high internal pressures and high temperature processing was proposed. This approach led to the development of regenerated gratings in standard photosensitive fibres with transmission rejection $>10 \% \mathrm{~cm}^{-1}$. and which can tolerate temperatures as high as $1295^{\circ} \mathrm{C}[18,19]$. The use of hydrogen was critical to obtain index modulation of useful magnitude for very high temperature operation, since it permitted enhanced localisation of the pressure differences between processed and unprocessed regions. However, in principle the model proposed in [19] can be independent of this and recent work demonstrates that this is so although for much lower temperature operation [20]. In this paper, we concentrate on studying the thermal properties of regenerated gratings for ultra high temperature operation and show complex behaviour until properly stabilised. There is a linear growth in grating strength with length. Further, we regenerate two types of complex structures (superposed twin grating and a Moirè grating) to demonstrate that all the properties introduced by the seed grating are retained with nanometer resolution, suggesting that this method could form an advanced processing method for creating holographic structures that go beyond simply 1-D filters and which have ultra-high thermal stability.

\section{REGENERATION}

\subsection{Fabrication of seed gratings}

In order to precipitate the structural change associated with regenerated gratings, a seed grating is necessary. In practice, we have confirmed that the stronger the seed grating, the stronger the final regenerated grating. For this case, a conventional Bragg grating inscribed into hydrogen loaded ( 24 hours, $200 \mathrm{~atm}, 70^{\circ} \mathrm{C}$ ) relatively highly germanium doped step index fibre with no boron $\left(r_{\text {core }} \sim 2 \mu \mathrm{m},\left[\mathrm{GeO}_{2} \sim\right.\right.$

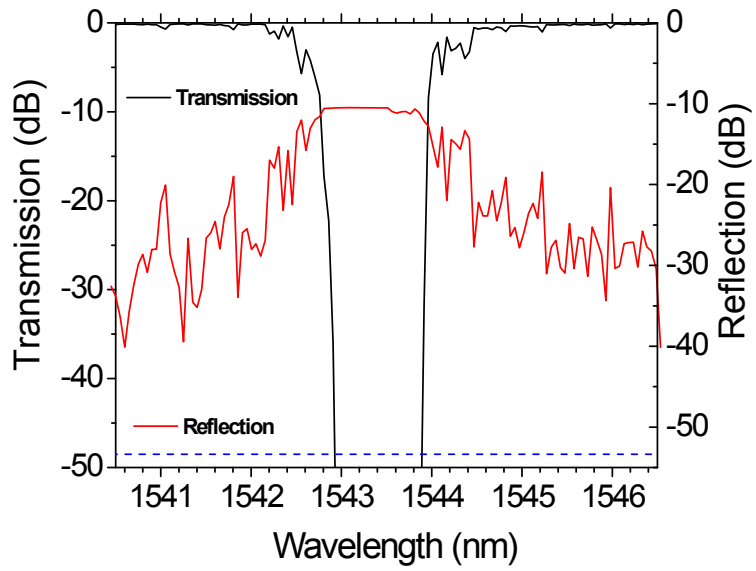

FIG. 1 Transmission and reflection spectra of the "conventional" seed grating. Noticeably, the large side lobes of this structure obscure the stitching errors expected from the phase mask used. The dashed line represents the noise floor.

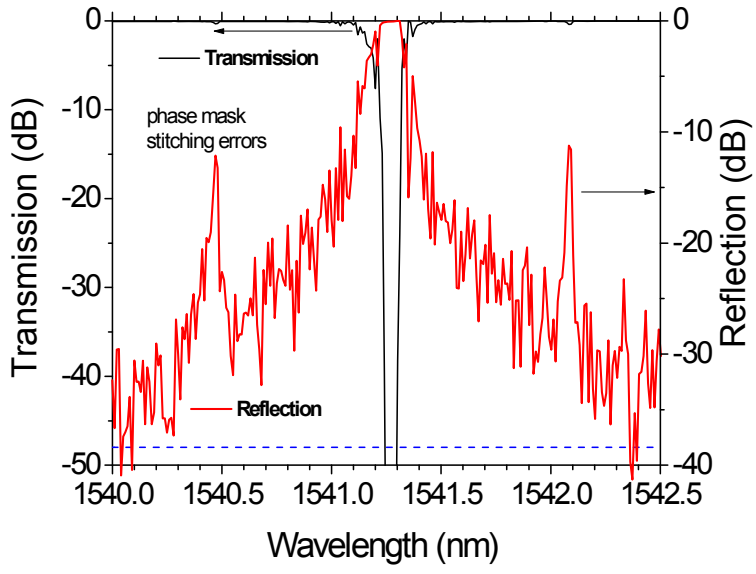

FIG. 2 Transmission and reflection spectra of the regenerated grating. Noticeably, the stitching errors of the phase mask are clearly visible indicating all the relative phase information has been retained. The dashed line represents the noise floor.

$10.5 \mathrm{~mol} \%$ ], $\left.\Delta n_{\mathrm{co} / \mathrm{cl}}=0.012\right)$, using the $244 \mathrm{~nm}$ output from a frequency doubled $\mathrm{Ar}^{+}$laser $\left(\mathrm{P} \sim 50 \mathrm{~W} / \mathrm{cm}^{2}, f_{\text {cumulative }} \sim\right.$ $(6-12) \mathrm{kJ} / \mathrm{cm}^{2}$, the same as that reported in $\left.[18,19]\right)$. Figure 1 shows the transmission and reflection of a very strong type I Bragg grating, readily exceeding the noise floor in transmission of our tunable laser and power meter setup (res: $1 \mathrm{pm}$ ). Ignoring the slight quadratic chirp in the transmission profile, the simulation spectra for a uniform grating, based on transfer matrix solution of the coupled mode equations, was fitted to the bandwidth to estimate the index modulation achieved: $\Delta n_{\text {mod }} \sim 1.6 \times 10^{-3}$, consistent with a grating $>120 \mathrm{~dB}$ in strength.

\subsection{Fabrication of regenerated gratings}

Using a processing procedure identical to that optimised in $[18,19]$, ultra strong seed gratings were thermally processed sequentially with a standard recipe. At $950^{\circ} \mathrm{C}$ the onset of regeneration is observed, and over time as the seed grating disappears completely, the regenerated grating appears. The final transmission and reflection spectra of the regenerated grating, obtained from the seed grating shown in Figure 1, are shown in Figure 2. It is more than $50 \mathrm{~dB}$ in strength 


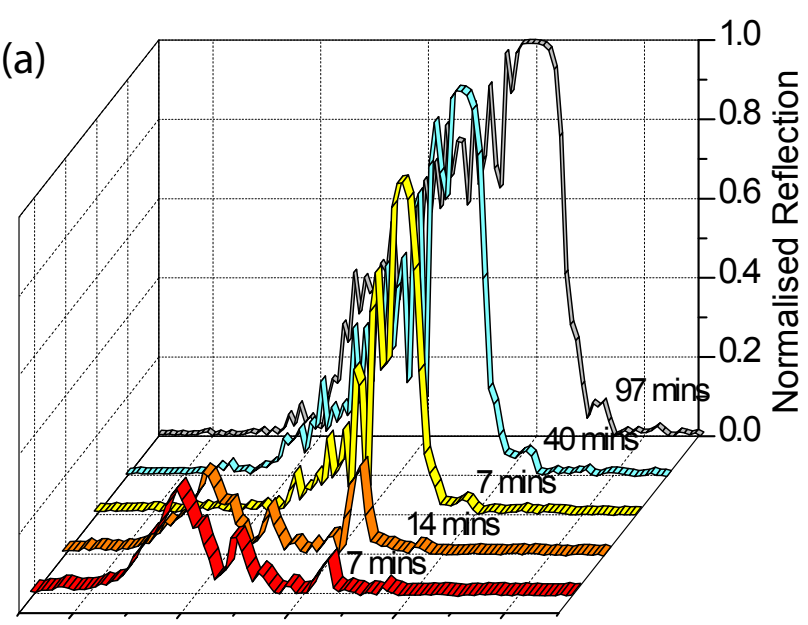

1552.61552 .81553 .01553 .21553 .4

Wavelength $(\mathrm{nm})$

(b)

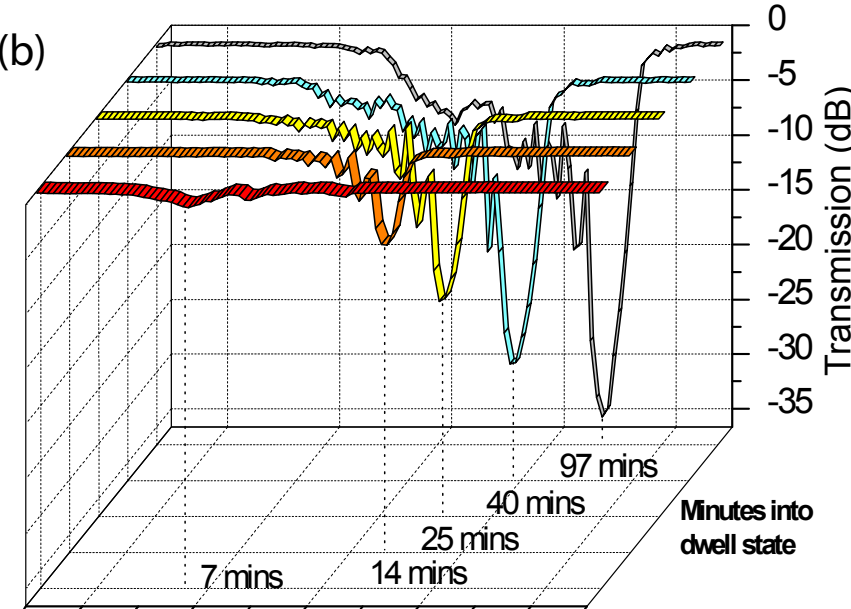

1552.61552 .81553 .01553 .21553 .4

Wavelength $(\mathrm{nm})$

FIC. 3 (a) Reflection (normalised) and (b) transmission spectra during formation and growth of regenerated grating. Significant, amplified quadratic chirp is observed.

and below the noise floor. Numerical simulation indicates an index modulation of $\Delta n_{\text {mod }} \sim 1.55 \times 10^{-4}$, which is substantial. However, the average index may likely be greater than this since the Bragg wavelength, $\lambda_{B}$, tends to be shifted longer wavelengths to that of the seed grating at room temperature, indicating that the fringe contrast is not optimal.

In order to study the growth and annealing properties, a second regenerated grating was made from a weaker seed grating, written with a cumulative fluence $\sim 30 \%$ less than that of the first grating, so that the full transmission spectrum can be observed within the noise floor of the tunable laser and power meter setup. Figure 3 shows a close-up of the grating formation over time at $\sim 950^{\circ} \mathrm{C}$. In this example, the structure is less uniform with a quadratic chirp present. This chirp is an exaggerated copy of the seed grating quadratic chirp so the complex profile of the seed grating was preserved. When tension is removed during regeneration, $\lambda_{B}$ is the same between seed and regenerated grating, also consistent with our previously reported observations $[18,19]$.
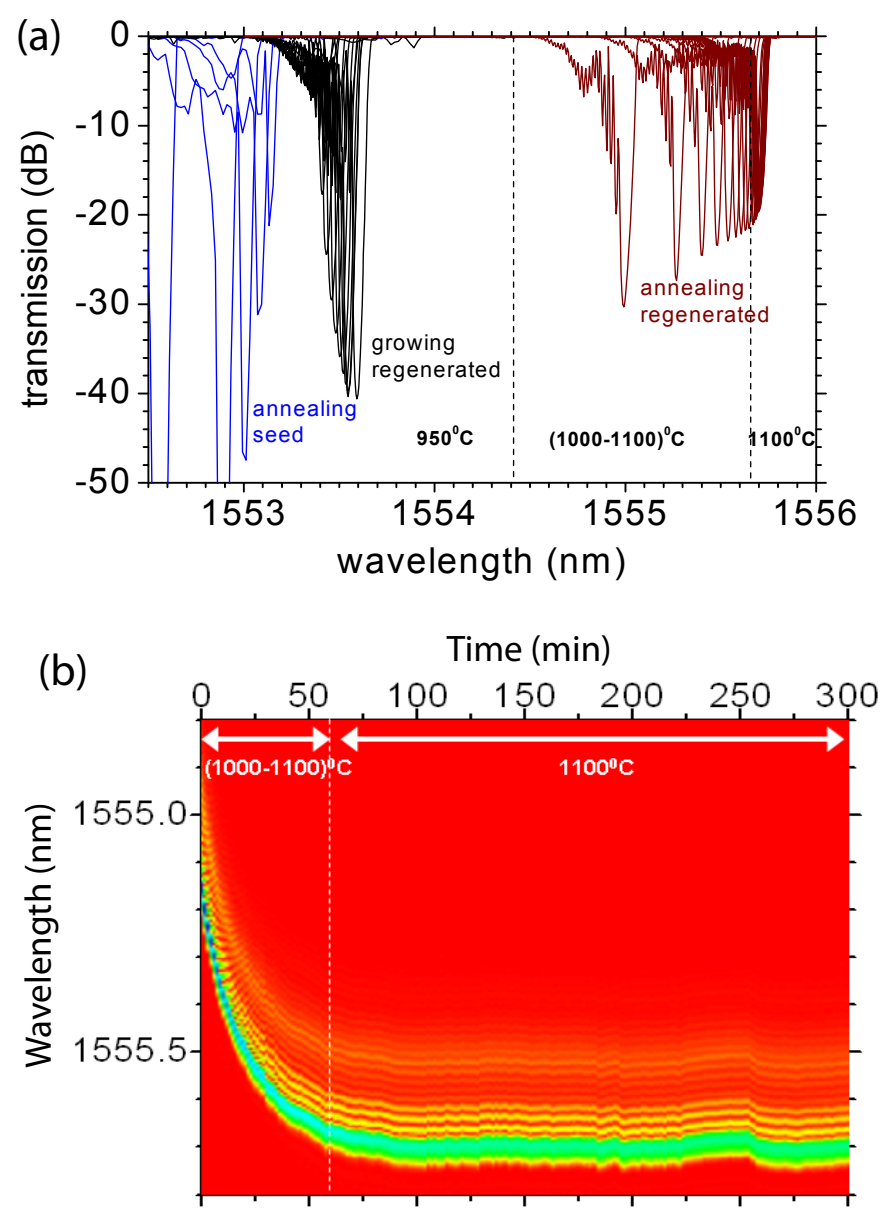

FIG. 4 (a) Summary of seed grating decay, regenerated grating formation and annealing; (b) detailed examination of the annealing of the grating between $1000^{\circ} \mathrm{C}$ and $1100^{\circ} \mathrm{C}$.

\section{ANNEALING STUDY}

The first regenerated grating, shown in Figure 1, was then cycled back to room temperature, back up to $1100^{\circ} \mathrm{C}$ and back to room temperature. For the short exposure times of $\sim 10 \mathrm{mins}$ at each temperature every $100^{\circ} \mathrm{C}$, no changes are observed in the grating spectra within the noise floor.

The second regenerated grating was used to determine the longer term performance at $1000^{\circ} \mathrm{C}$ and $1100^{\circ} \mathrm{C}$. The regenerated grating, $\sim 42 \mathrm{~dB}$ in strength, was cooled back to $\sim 200^{\circ} \mathrm{C}$ before being taken up to $1000^{\circ} \mathrm{C}$ to show that no decay occurs at lower temperatures. The grating was then ramped to $1100^{\circ} \mathrm{C}$ over $\sim 50$ minutes. It was then allowed to sit at $1100^{\circ} \mathrm{C}$ for an additional 4 hours and 10 minutes. During the ramping period the grating decays with a single exponential to $\sim 19 \mathrm{~dB}$. This stabilised to a final rejection of $\sim 18 \mathrm{~dB}$ within ten minutes at $1100^{\circ} \mathrm{C}$. The grating strength after this point remained constant over the remaining exposure period. Figure 4(a) shows a full summary of seed grating decay, regeneration formation and then the annealing results whilst Figure 4 (b) shows a contour image diagram of the decay process over time showing the Bragg wavelength shift with temperature, the exponential decay in contour form and the subsequent stable and steady performance after an hour. When comparing with our previous work, we have shown that by simply extending the length of the seed grating we can regen- 
erate longer gratings that increased in strength approximately linearly after stabilisation. In the previous work, the regenerated grating was reported to be $\sim 2.3 \mathrm{~dB}$ over $0.5 \mathrm{~cm}$, or a coefficient of $4.6 \mathrm{~dB} / \mathrm{cm}$ within the same fibre used here and for the same seed grating writing conditions. The reported value for $5 \mathrm{~cm}$ in this work after stabilisation is $\sim 18 \mathrm{~dB}$, less than the $23 \mathrm{~dB}$ expected; this is because the seed grating fluence of this grating was actually less than that used previously so as to be able to observe the peak regeneration, $\sim 42 \mathrm{~dB}$, prior to stabilisation. (The first grating broke during experiments so additional stabilisation could not be pursued). Within experimental error, there is no evidence to suggest that a non-linear regenerated grating strength with length in this fibre.

The observation that the regenerated grating is stabilised after an initial decay process suggests two contributions to the grating strength, the second contribution clearly enabling extremely strong thermal resistance of the grating (up to $1295^{\circ} \mathrm{C}$ as demonstrated in [19]). The single exponential process supports the notion that the first decay process is indeed a singular one. It too is quite stable after formation at least at temperatures below the regeneration temperature of $\sim 950^{\circ} \mathrm{C}$. Given the localisation of the changes to the periodic scale of the seed grating, the likely contributions are related to stresses not only at the core-cladding interface but indeed between processed regions. What is important to note is that the decrease in grating strength does not appear to be accompanied by any average index decrease (i.e. $\lambda_{B}$ continues to be red-shifted and no blue shift is observed though its difficult to separate this because of the slow thermal equilibration of our oven. Based on this observation, the appearance of two contributions may be an artifact of gradual fringe relaxation until the one contribution is stabilised and this suggests there is a longitudinal component (through stress between processed and unprocessed regions).

With regards to the mechanism of formation, we have ruled out the role of fluorine in the cladding in this process by observing nearly identical regeneration and annealing within fibres that have no fluorine at all. Therefore, the presence of any fluorine diffusion, thought to be critical in [15], would likely be largely coincidental in regeneration (though the presence of F may change the initial seed state). The regenerated grating strength obtained is shown to be determined by a number of factors including seed grating strength and as well numerical aperture (NA) determined predominantly by the concentration of $\mathrm{GeO}_{2}$ within these fibres. This is consistent with an interface driven process of structural change as suggested in $[18,19]$. When a fibre which has 3 times the concentration of $\mathrm{GeO}_{2}$ than the fibre used here, but is loaded with $\mathrm{B}_{2} \mathrm{O}_{3}$; which is known to often reduce stresses in germanosilicate fibres at elevated temperatures. In order to reduce its numerical aperture below this, we find the regenerated grating strength is less than half. Therefore, the regenerated grating appears to have a dependency on stress changes both longitudinally and transversally (and therefore circumferentially). More detailed scientific studies are currently underway to explore the changes involves.

\section{REGENERATION OF COMPLEX GRATINGS}

In order to determine whether this process can be applied beyond simple Bragg grating writing as a realistic approach to the production of complex gratings and patterns and structures that can operate at high temperature whilst retaining the complexity of a nano-scaled device, we explored the impact of the regeneration process on two complex grating structures: (1) a structure consisting of two superposed gratings with $\lambda_{1} \sim 1548.73 \mathrm{~nm}$ and $\lambda_{2} \sim 1553.56 \mathrm{~nm}$, i.e. with $\Delta \lambda \sim$ $4.8 \mathrm{~nm}$; and (2) a dual channel grating produced by writing a Moiré grating. In a Moiré grating, the refractive index variation along the length of the grating is also different where a uniform period, $\Lambda_{B}$, is modulated by a low spatial frequency sinusoidal envelope of period, $\Lambda_{e}$, that produce two sidebands (essentially a phase shifted structure built up from a periodic distribution of identical phase shifts). Given the sensitivity of the Moirè grating to any perturbation in phase, the preservation of the transmission notch and overall profile will be indicative of nanoscale resolution in the regenerated structure.

For the superposed gratings ( $\mathrm{L} \sim 5 \mathrm{~mm}$ ) were inscribed into a $\mathrm{H}_{2}$ loaded ( 24 hours, $P=100 \mathrm{~atm}, \mathrm{~T}=100^{\circ} \mathrm{C}$ ) $\mathrm{GeO}_{2}$ doped core silica fibre $\left(\left[\mathrm{GeO}_{2}\right] \sim 10 \%\right.$, fabricated at CGCRI) using a pulsed $\mathrm{KrF}$ exciplex laser $(248 \mathrm{~nm}$, pulse duration $=20 \mathrm{~ns}$, $f_{\text {pulse }} \sim 70 \mathrm{~mJ} / \mathrm{cm}^{2}$, repetition rate $=200 \mathrm{~Hz}$ ). The Moiré grating was written into a fibre which was similar to that used for the superposed grating but also had boron to increase the seed photosensitivity. Regeneration is carried out with an identical recipe to that described earlier but inside a short fibre microheater. The hot zone of this heater is supposedly uniform over $5 \mathrm{~mm}$ only (the exact variation along this length is not known but we suspect a Guassian profile), and this dictates the grating length.

\subsection{Superposed gratings}

Sample \#1 was prepared by superposing two seed gratings with Bragg wavelengths $\lambda_{1} \sim 1548.73 \mathrm{~nm}$ and $\lambda_{2} \sim 1553.56 \mathrm{~nm}$, i.e. with $\Delta \lambda \sim 4.8 \mathrm{~nm}$. Each of the seed gratings was of moderate strength with transmission loss at $\lambda \sim-20 \mathrm{~dB}$ (grating with $\lambda_{1}$ being slightly stronger than that at $\lambda_{2}$ ). The superposition of two gratings leads to a compound form of the local index modulation described as [22],

$$
\begin{aligned}
& \Delta n(z)=2 \Delta n_{0} \cos \left(\frac{\pi\left(2 \Lambda_{B 1}+\Delta \Lambda\right)}{\Lambda_{B 1}\left(\Lambda_{B 1}+\Delta \Lambda\right)} z+\frac{\Delta \Phi}{2}\right) \\
& \times \cos \left(\frac{\pi \Delta \Lambda z}{\Lambda^{2}}-\frac{\Delta \Phi}{2}\right) .
\end{aligned}
$$

$\Lambda_{B 1}$ and $\Lambda_{B 2}$ are the periods of the gratings with $\Lambda_{B 2}=$ $\Lambda_{B 1}+\Delta \Lambda$ and $\Delta \Phi$ is the initial phase difference of the gratings. It is clear from this expression any non-uniformity introduced by the thermal annealing process will result in a spread of $\Delta \Phi$ and broadening of the peaks. The structure was then thermally processed as described earlier until regeneration was complete. The results are summarised in Figure 5. Within experimental uncertainty, the Bragg wavelength separation remains the same $(\sim 4.8 \mathrm{~nm})$ although, as expected the annealing has led to a decrease in average index so that 

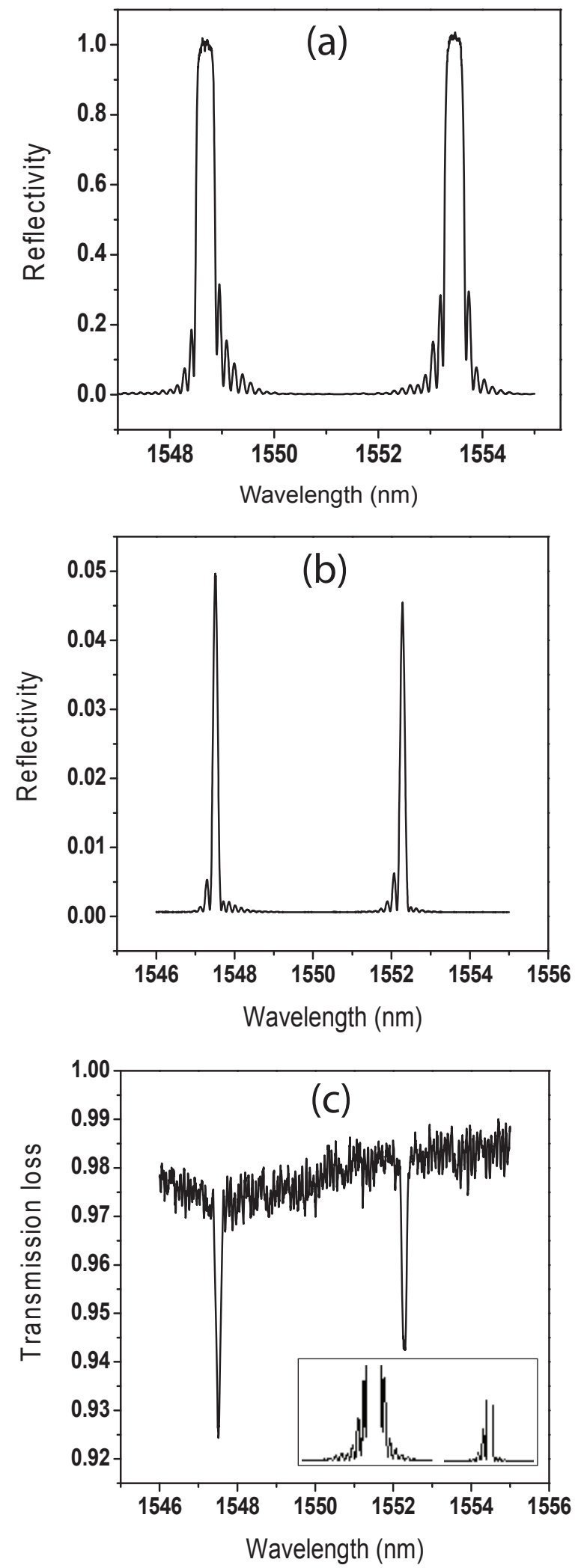

FIG. 5 Spectrum of dual over-written gratings. (a) Normalised reflection spectrum of the seed; (b) and (c) reflection and transmission spectrum of the regenerated grating respectively represented in absolute scale. Inset: close-up of side lobe structure of right hand peaks of seed and regenerated grating for comparison.

the Bragg wavelengths are blue-shifted. This reduction leads to a change in the phase distribution and the regenerated gratings have a more asymmetric profile, shown in the inset of Figure 5(c). This is consistent with a very weak Gaussian chirp on the grating. The origin for this chirp almost certainly

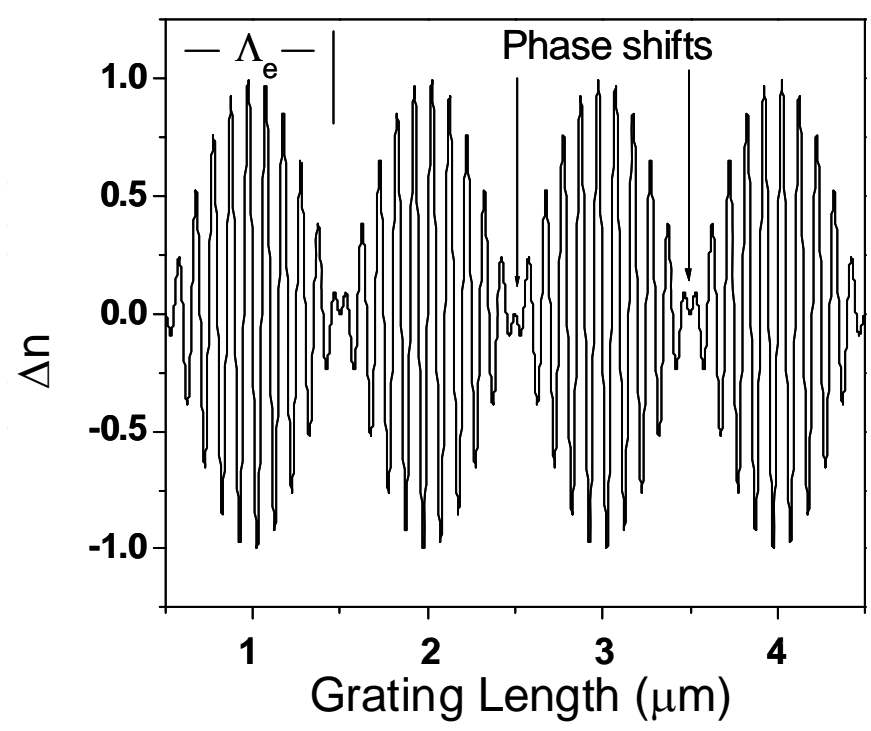

FIG. 6 Index modulation introduced into the seed Moiré grating.

arises from the hot zone temperature distribution of the microheater rather than any intrinsic grating property.

\subsection{Moiré gratings}

In a Moiré grating, a uniform period, $\Lambda_{B}$, is modulated by a low spatial frequency sinusoidal envelope of period, $\Lambda_{e}$, (see Figure 6) that produces two sidebands. The structure is equivalent to two gratings with stopgaps that overlap sufficiently to produce a resonant phase shift-like structure in the stop gap of the superstructure. A similar profile is obtained by placing phase shifts with a low frequency period along a uniform grating. The position dependent index amplitude modulation profile can be described as [23]:

$$
\Delta n(z)=2 n \Delta n_{0} F(z) \cos \left(\frac{2 \pi N z}{\Lambda_{B}}\right) \cos \left(\frac{2 \pi M z}{\Lambda_{e}}\right)
$$

where $N$ and $M$ are integers and $2 n \Delta n_{0}$ is the UV induced index change, $F(z)$ is the apodisation profile. On simplifying, Eq. (2) directly leads to the resultant spatial frequencies at the sum and difference frequencies where two Bragg reflections will occur and may be represented as

$$
\begin{aligned}
\Delta n(z)=n \Delta n_{0} F(z)\left\{\cos \left(\frac{2 \pi N}{\Lambda_{B}}\left[1+\frac{M \Lambda_{B}}{N \Lambda_{e}}\right] z\right)\right. \\
\left.+\cos \left(\frac{2 \pi N}{\Lambda_{B}}\left[1-\frac{M \Lambda_{B}}{N \Lambda_{e}}\right] z\right)\right\}
\end{aligned}
$$

The new reflection has two effective bands separated in wavelength, $\Delta \lambda$, as,

$$
\Delta \lambda=\frac{\lambda_{B}^{2}}{2 n_{\mathrm{eff}} \Lambda_{e}} .
$$

Based on the principle mentioned above a dual seed grating with a $100 \mathrm{GHz}$ separation, i.e. $\Delta \lambda \sim 0.8 \mathrm{~nm}$ was written. Selected $\Lambda_{B}=533.17 \mathrm{~nm}$ produces a grating with $\lambda_{B} \sim$ $1554 \mathrm{~nm}$. Modulating $\Lambda_{B}$ with $\Lambda_{e}=1028 \mu \mathrm{m}$ we could generate two channels Bragg wavelengths, $\lambda_{1}=1553.51 \mathrm{~nm}$ and $\lambda_{2}=1554.34 \mathrm{~nm}$ respectively. The effective refractive index of the fibre is $n_{\text {eff }}=1.4573$. A precisely controlled scanning beam writing setup was used to produce $\pi$-phase shifts at specific 
locations of the grating to generate the required low frequency sinusoidal modulation of the index profile. A summary of the induced profile is shown in Figure 6. The seed grating reflection profile is shown in Figure 7(a) and the regenerated grating reflection and transmission profiles are shown in Figures 7(a) and 7(c). Unlike the superposed gratings, where the sidebands of the grating are a result of the interference between end reflections of the grating and therefore susceptible to temperature gradients in the micro heater hot zone, the interference in the phase shift region is a result of the distributed interference between the grating and super period of the phase shifts. This means the structure is less sensitive to overall gradients on a macro scale. Importantly, the interference in the phase shift region is preserved after regeneration indicating that despite the very large macro heating process involved in creating the regenerated grating, the structure retains full memory of the seed grating, indicating that there is no evidence of a diffusive process that would alter the phase relationship anywhere over the grating length. Full preservation on a nanoscale is maintained through regeneration and this is a remarkable result.

\section{CONCLUSION}

Strong regenerated gratings $(\sim 18 \mathrm{~dB}, \mathrm{~L}=5 \mathrm{~cm})$ that can withstand temperatures in excess of $1100^{\circ} \mathrm{C}$ have been produced. Further, complex regenerated gratings $(\mathrm{L}=0.5 \mathrm{~cm})$ were demonstrated. Two dual channel filter designs; a superposed grating and a Moiré grating which were both fabricated with more than $4 \%$ transmission. The regenerated superposed structure showed signs of a small chirp possibly arising from the slightly Gaussian profile of the micro heater hot zone employed. This suggests that regenerated gratings can be thermally post-tailored during regeneration from the seed grating on a macro scale. In contrast, despite the significantly reduced strength and the reduced average index change (measured as a shift to shorter wavelengths) the regenerated Moiré grating exactly preserved the interference profile within the central transmission notch of the grating spectrum, and therefore the embedded phase information of the seed grating. Given that much of the general process involves glass re-quenching under a different thermal history, the processes described here are unlikely to be confined to silica fibres loaded with or without hydrogen (as recent results indicate). Rather, the regeneration process has huge scope to be applied to many numerous materials systems. It opens the way of using nanoscale precision laser processing to introduce nanoscale patterns and structures in materials which can then be thermally processed, with unique recipes given each environment, for additional stability. We believe this has the potential to greatly expand advanced holographic processing of systems and templates by extending the lifetime and operational thresholds of the materials to a level not previously thought possible.

\section{ACKNOWLEDGEMENTS}

Funding from the Australian Research Council (ARC) and an International Science Linkage Grant from the Department of Industry, Innovation, Science and Research (DIISR), Australia
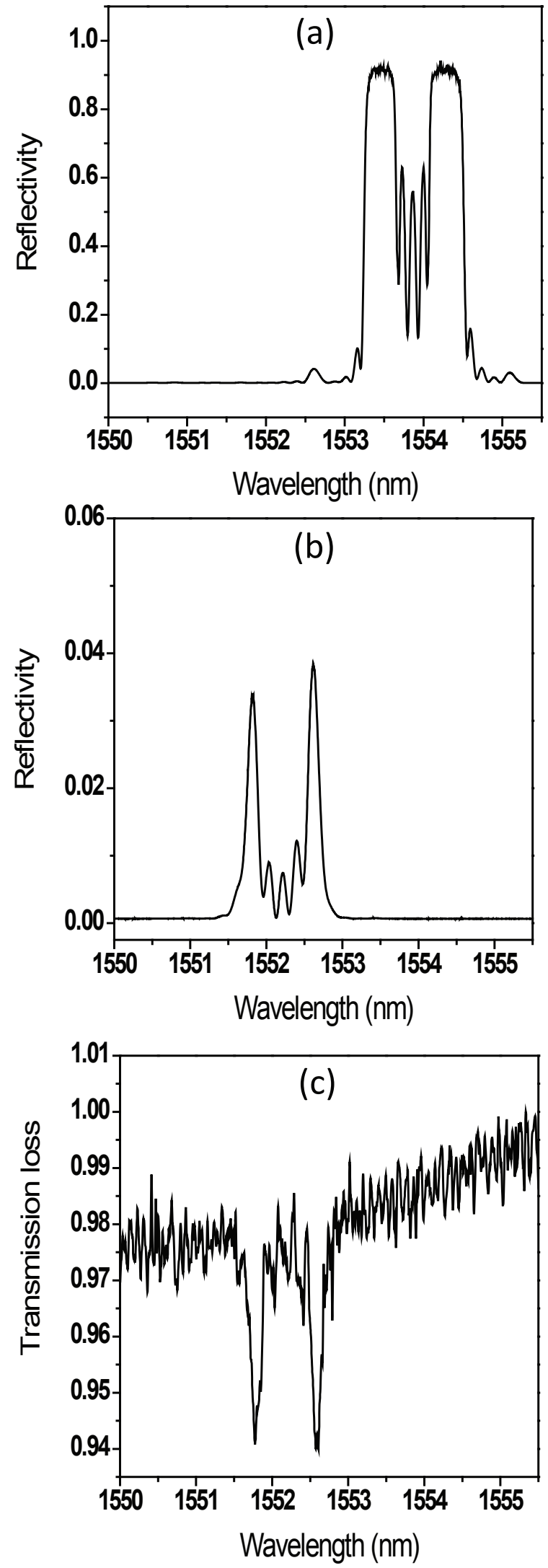

FIC. 7 Spectrum of Moiré grating. (a) Normalised reflection spectrum of the seed, (b) and (c) reflection and transmission spectrum of the regenerated grating respectively represented in absolute scale.

and the Council of Scientific and Industrial Research (CSIR), India, under the $11^{\text {th }}$ five year plan is acknowledged. 


\section{References}

[1] R. J. Schroeder, T. Yamate, and E. Udd, "High pressure and temperature sensing for the oil industry using fibre Bragg ratings written into side hole single mode fibre" Proc. SPIE 3746, 42-45 (1999).

[2] A. D. Kersey, "Optical fiber sensors for permanent downwell monitoring in the oil and gas industry" IEICE T. Electron. 83, 400-404 (2000).

[3] J. C. Cardozo da Silva, C. Martelli, H. J. Kalinowski, E. Penner, J. Canning, and N. Groothoff, "Dynamic analysis and temperature measurements of concrete cantilever beam using fibre Bragg gratings" Opt. Laser Eng. 45, 88-92 (2007).

[4] Y. Shen, J. He, Y. Qiu, W. Zhao, S. Chen, T. Sun, and K. T. Grattan, "Thermal decay characteristics of strong fiber Bragg gratings showing high temperature sustainability" J. Opt. Soc. Am. B 24, 430-438 (2007).

[5] 0. V. Butov, E. M. Dianov, and K. M. Colant, “Nitrogen-doped silica core fibres for Bragg grating sensors operating at elevated temperatures" Meas. Sci. Technol. 17, 975-979 (2006).

[6] M. Aslund, and J. Canning, "Annealing properties of gratings written into UV-presensitized hydrogen-out diffused optical fiber" 0pt. Lett. 25, 692-694 (2000).

[7] J. Canning, K. Sommer, and M. Englund, "Fibre gratings for high temperature sensor applications" Meas. Sci. Technol. 12, 824-828 (2001).

[8] W. X. Xie, P. Niay, P. Bernage, M. Douay, J. F. Bayon, T. Georges, M. Monerie, and B. Poumellec, "Experimental evidence of two types of photorefractive effects occurring during photo inscriptions of Bragg gratings within germanosilicate fibers" Opt. Commun. 104, 185-195 (1993).

[9] L. Dong, W. F. Liu, and L. Reekie, "Negative index gratings formed by 193 nm laser" Opt. Lett. 21, 2032-2034 (1996).

[10] N. Groothoff, and J. Canning, "Enhanced type IIA gratings for hightemperature operation" Opt. Lett. 29, 2360-2362 (2004).

[11] J. L. Archambault, L. Reekie, and P. St. Russell, "100\% reflectivity Bragg reflectors produced in optical fibres by single excimer laser pulses" Electron. Lett. 29, 453-455 (1993).
[12] P. Hill, G. R. Atkins, J. Canning, G. Cox, and M. G. Sceats, "Writing and visualisation of low threshold type II Bragg gratings in stressed optical fibres" Appl. Opt. 33, 7689-7694 (1995).

[13] D. Grobnic, C. W. Smelser, S. J. Mihailov, and R. B. Walker, "Long term thermal stability tests at $1000^{\circ} \mathrm{C}$ of silica fibre Bragg gratings made with ultrafast laser radiation" Meas. Sci. Technol. 17, 10091013 (2006).

[14] J. Canning, "Fibre gratings and devices for sensors and lasers" Laser Photonics Rev. 2, 275-289 (2008).

[15] M. Fokine, "Formation of thermally stable chemical composition gratings in optical fibers" J. Opt. Soc. Am. B 19, 1759-1765 (2002).

[16] S. Trpkovski, D. J. Kitcher, G. W. Baxter, S. F. Collins, and S. A. Wade, "High temperature-resistant chemical composition gratings in $\mathrm{Er}^{3+}$-doped optical fiber" Opt. Lett. 30, 607-609 (2005).

[17] B. Zhang, and M. Kahriziet, "High temperature resistance fiber Bragg grating temperature sensor fabrication" IEEE Sens. J. 7, 586590 (2007).

[18] S. Bandyopadhyay, J. Canning, M. Stevenson, and K. Cook, "Ultrahigh-temperature regenerated gratings in boron-codoped germanosilicate optical fiber using 193 nm" Opt. Lett. 33, 19171919 (2008).

[19] J. Canning, M. Stevenson, S. Bandyopadhyay, and K. Cook, "Extreme silica optical fibre gratings" Sensors 8, 6448-6452 (2008).

[20] E. Linder, C. Chojetski, S. Brueckner, M. Becker, M. Rothhardt, and $\mathrm{H}$. Bartelt, "Thermal regeneration of fibre Bragg gratings in photosensitive fibres" Opt. Express 17, 12523-12531 (2009).

[21] K. W. Raine, R. Feced, S. E. Kanellopoulos, and V. A. Handerek, "Measurement of axial stress at high spatial resolution in ultraviolet-exposed fibers" Appl. Opt. 38, 1086-1095 (1999).

[22] J. Bao, X. Zhang, K. Chen, and W. Zhou, "Spectra of dual overwritten Bragg grating" Opt. Commun. 188, 31-39 (2001).

[23] M. Ibsen, M. K. Durkin, and R. I. Laming, "Chirped Moiré fiber gratings operating on two wavelength channels for use as dualchannel dispersion compensators" IEEE Photonic. Tech. Lett. 10, 84-86 (1998). 